\title{
High repetition rate Petawatt lasers
}

\author{
Luis Roso* \\ Center for Pulsed Lasers, CLPU, Villamayor, Salamanca, Spain.
}

\begin{abstract}
Petawatt lasers are now available in a number of facilities around the world and are becoming a very useful tool in physics and engineering. Some of such lasers are able -or will be able soon- to fire at high repetition rates (one shot per second or more). Experiments at such repetition rates have certain peculiarities that are to be briefly exposed here, based on the author's experience with the Salamanca VEGA-3 laser. VEGA-3 is a $30 \mathrm{fs}$ PW laser, firing one shot per second.
\end{abstract}

\section{Introduction}

Laser acceleration of electrons and protons is now a reality at low repetition rate. However there are many lasers troughout the world beyond $100 \mathrm{TW}$ and with repetition rates around one shot per second, or more. High repetition rate lasers are now under consideration, $\mathrm{kHz}$ at the $10 \mathrm{TW}$ level and $100 \mathrm{~Hz}$ at the $100 \mathrm{TW}$ level and $10 \mathrm{~Hz}$ at the PW level. Those systems represent a major technological revolution so far. A lot of problems have been solved or are under solution regarding those systems, as cryo-cooling, large crystal growing, large diffraction gratings construction, transverse lasing, big femtosecond broadband mirrors, pumping lasers, and many more. However one point is underdeveloped so far: targetry (with associated shot to shot diagnostic). This is going to be analyzed in the present contribution.

Table 1. Basic parameters of the VEGA laser system installed at CLPU. It is a Ti:Sapphire laser emitting around $800 \mathrm{~nm}$. Pulse duration is $30 \mathrm{fs}$ in all three lines.

\begin{tabular}{|l|c|c|c|}
\hline & $\begin{array}{c}\text { Energy } \\
\text { per shot }\end{array}$ & $\begin{array}{c}\text { Peak } \\
\text { Power }\end{array}$ & $\begin{array}{c}\text { Repetition } \\
\text { rate }\end{array}$ \\
\hline VEGA 1 & $0.6 \mathrm{~J}$ & $20 \mathrm{TW}$ & $10 \mathrm{~Hz}$ \\
\hline VEGA 2 & $6 \mathrm{~J}$ & $200 \mathrm{TW}$ & $10 \mathrm{~Hz}$ \\
\hline VEGA 3 & $30 \mathrm{~J}$ & $1 \mathrm{PW}$ & $1 \mathrm{~Hz}$ \\
\hline
\end{tabular}

\section{Present day technology}

Recent advances in Chirped Pulse Amplification (CPA) allowed dramatic improvements of the laser peak power. CPA was developed in mid eighties at the University of Rochester. After the initial paper of Donna Strickland and Gerard Mourou [1], the technique was rapidly developed [2]. CPA consists in lengthening the duration of the pulse being amplified in a reversible fashion. The reversibility, or ability to recompress the pulse after amplification relies on the technique of optical pulse compression developed by Treacy [3] and Martínez [4]. By lengthening the pulse in time, energy can be efficiently extracted from the laser gain medium, avoiding at the same time damage to the laser amplifier components (typically Ti:Sapphire or other doped crystals). CPA is particularly useful for efficient utilization of solid-state laser media with high stored energy density $(1 \mathrm{~J} / \mathrm{cm} 2)$, where full energy extraction in a short pulse would lead to intensities above the damage threshold of the amplifier materials.

During more than three decades CPA has evolved and is now a developed technology with many different directions of evolution. The frontier land now is the 1 to $10 \mathrm{PW}$ peak power range. It is well know that there are several possibilities to arrive to the PW peak power. One Megajoule shots with duration of one nanosecond correspond to one PW, however this limit has not been achieved so far. There are two stadium-size lasers in the world, the National Ignition Facility [5] at Livermore, California, USA, and the Laser MegaJoule [6] at Bordeaux, France. Both arrive to the Megajoule per shot but combing incoherently many beams.

One kilojoule shot with duration of one picosecond corresponds to one PW. This limit has been achieved so far. One very representative example is the PETAL laser (adjacent to the French Laser MegaJoule). PETAL was the first kilojoule-class laser to arrive to $1.2 \mathrm{PW}$ peak power. Of course these lasers work at single shot regime, and they need hours from shot to shot.

The intensity limit corresponds to PW's of few femtoseconds. With conventional CPA technology the limit -at those amplification limits is $23-30 \mathrm{fs} .30 \mathrm{~J} / 30 \mathrm{fs}$ correspond to one PW. With OPCPA (Optical Parametric CPA) it seems feasible to arrive to $15 \mathrm{fs}$ outpout, and $15 \mathrm{~J} / 15$ fs correspond to one PW also. In this contribution we will refer to those systems as intensity PW (we call them high intensity PW's because the wavefront quality is much better that for the kilojule 
or Megajoule lasers and then it is possible to focus them to extreme intensities. The possibility to go to much shorter durations ( $5 \mathrm{~J} / 5 \mathrm{fs}$ ) at the PW peak power level is under consideration. Sub fs PW are not feasible for the moment.

Today, arriving to the PW level is more or less the state of the art of the reliable technology. Several projects to arrive to the next step -10 PW- are well advanced particularly for intensity lasers. At the time of writing the two leading systems are at the Shanghai SuperintenseUltrafast Lasers Facility, SULF, Shanghai [7] and at the Center for Relativistic Laser Science, Institute for Basic Science, Gwangju, South Korea [8] both close to 5 PW, while $10 \mathrm{PW}$ systems are under construction in the Extreme Laser Infrastructure ELI,

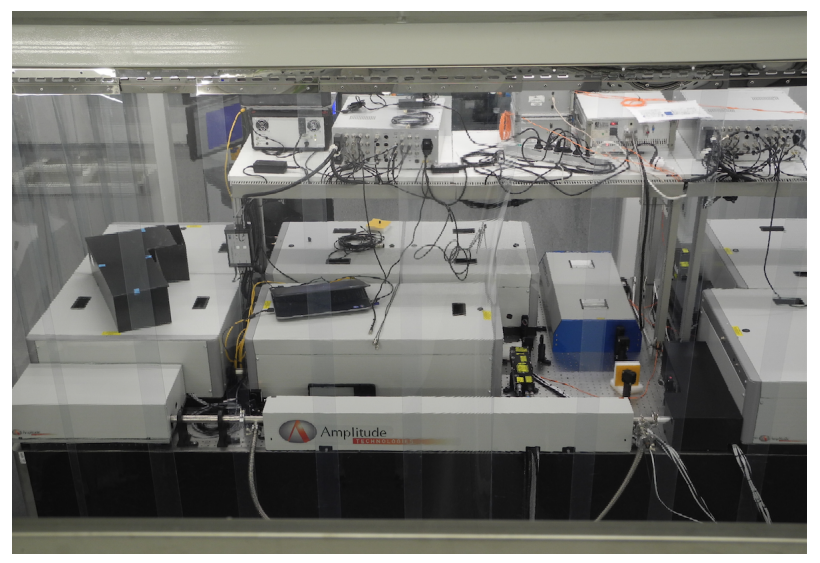

Fig. 1. General view of the VEGA laser front end, with the XPW system in front. XPW helps to get a high contrast of the pulse, something very necessary for solid targets.

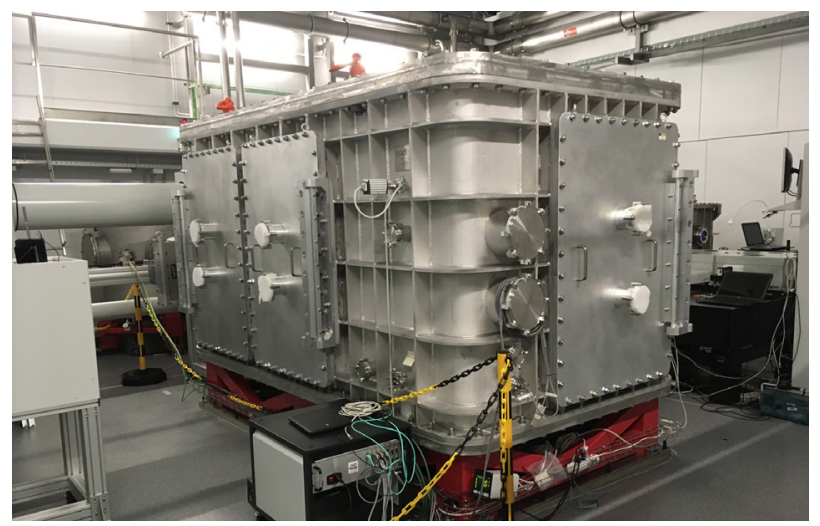

Fig. 2. VEGA-3 (the PW laser) compressor installed at Salamanca. The four gratings are at vacuum $\left(10^{-6} \mathrm{mbar}\right)$ and their vibrational stability is guaranteed by an optical table bound directly to the ground trough legs and bellows that are decoupled from the main vacuum vessel.

\section{High repetition rates}

One of the advantages of the development of the CPA techniques is the possibility of high repetition rates. The first Petawatt lasers were single shot systems with a long time for cooling between shot and shot. Today if we want a laser accelerator able to be compared to conventional accelerators, we need to work to increase the repetition rate.

Repetition rate at Megajoule or kilojoule levels is not under consideration because the huge pumping lasers they have, that require very long cooling times (from several minutes to hours). For the intensity PW's the situation is much different.

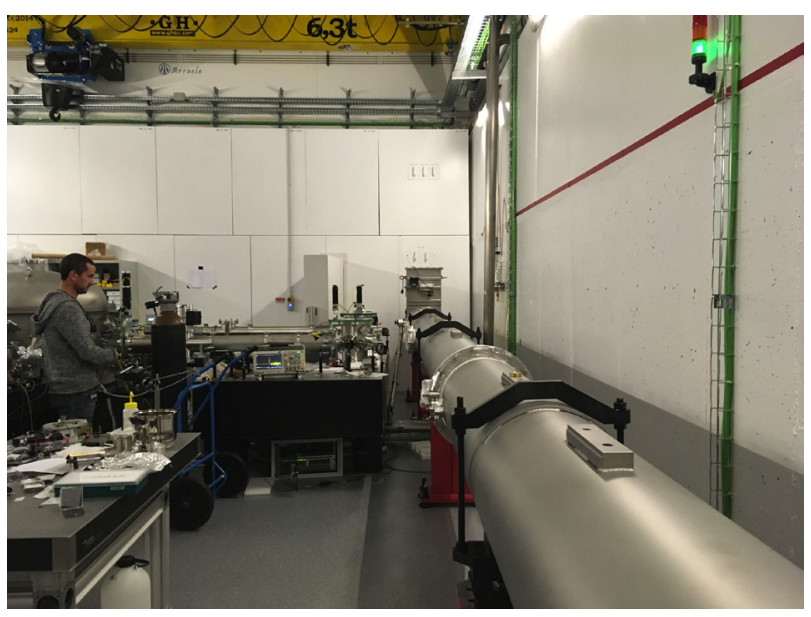

Fig. 3. Beam transport from the $\mathrm{PW}$ compressor to the experimental station. The beam diameter of VEGA-3 is $24 \mathrm{~cm}$ flat-top. The tubes are almost $50 \mathrm{~cm}$ in diameter

High repetition rate implies certain technological problems in the cooling of the different components. Among those problems, we must mention laser pumping, amplifiers cooling and damage of the optical elements. Price is also a major problem, but we are not going to comment on that.

The most relevant problem is the pumping system. For example we can comment on the Ti:Sapphire laser, the most common one in those lasers. Ti:Sapphire is a vibronic laser that has to be pumped by a blue-green laser with pulses around $10 \mathrm{~ns}(\mathrm{Q}$-switched). The most common pump is $\mathrm{Nd}$ :glass or $\mathrm{Nd}$ :solid. $\mathrm{Nd}$ :glass is the best and cheapest option for low repetition rate. But the duty cycle of the glass cooling prevents going beyond a few shots per minute. Nd:solid, typically Nd:YAG, is a good option for systems in the $0.1-100 \mathrm{~Hz}$ regime. However the problem in turn is the system to provide energy to the laser used to pump Ti:Sapphire. For high energies (beyond Joule) Nd:YAG is nowadays pumped by flash lamps and those lamps have a practical limit at $10 \mathrm{~Hz}$. The future is diode lasers. Diode pumped Nd:YAG are the best solution now below $10 \mathrm{TW}$ and will become the main future option as the diode price drops. Diode pump opens the door to Nd:YAG lasers at $100 \mathrm{~Hz}$ or even at $\mathrm{kHz}$ (although in this case the best option is $\mathrm{Nd}$ :YLF) and soon will allow a generation of extreme high repetition rate lasers [9].

Another possibility that is becoming real is the direct diode pumping of Ti:Sapphire without the need to use $\mathrm{Nd}$ :YAG doubled in frequency. In this case blue diodes are necessary (this is not a problem now) but also $10 \mathrm{~ns}$ pulse durations (and this is the main problem so far) [10]. 
Another very relevant problem is the cooling of the amplifiers, particularly the last amplifiers in the chain. The quantum efficiency of the Ti:Sapphire laser is not good. We pump it with $532 \mathrm{~nm}$ light and we get laser light around $800 \mathrm{~nm}$, this means that approximately one third of the pumping energy is lost. But not all pumping photons are used to generate laser photons, a percent of them is also lost. As a result more than 40 percent of the energy ends heating the amplifier crystal. Sapphire is a crystal very resistant mechanically and thermally, so the heating does not represent a structural problem. However a small change in the temperature ends in a local change of the refractive index, and therefore the energy lost heats locally the crystal and changes the light propagation through it. This is a major problem that can ruin the lasing capacity of any multi-pass amplifier. The way to solve this is to cool down the amplifier crystal. The cooling helps to the temperature homogeneity and moreover helps to distribute the dissipated heat throughout all the crystal because the thermal conductivity of materials as Ti:Sapphire increases as the temperature goes down.

A third important problem relevant enough to be mentioned here is the heat dissipation at the gratings and third quick damage in those cases. Damage threshold of today's gratings requires fluences not greater that 100 $\mathrm{mJ} / \mathrm{cm} 2$ (for $30 \mathrm{fs}$ pulse durations). For example this implies that the beam size of the VEGA PW is $24 \mathrm{~cm}$ flat top (that corresponds to $450 \mathrm{~cm} 2$, with an uncompressed beam around $45 \mathrm{~J}$ and a compressed beam around $30 \mathrm{~J}$ ). The diameter of the beam transport can be seen in Fig. 3. Reducing the beams size would require gratings more resistant to damage. Of course this is more relevant as the repetition rate increases. If gratings were to close to the damage thresholds they could be specified for a few ten thousand shots. At one shot per hour this can be reasonable. With a shot per second this imply an impossible replacement of the gratings every day!

With all this in mind, a multi-Hz multi-PW system seems quite possible now. At the moment of writing there are only three lasers at the PW peak power level and at the $\mathrm{Hz}$ repetition rate operative in the World. The first in being operative was the BELLA laser system [11] at Lawrence Berkeley National Laboratory in California USA. The second one is the DRACO laser system [12] laser system at Dresden, Germany, and the third one is the VEGA laser system [13] at Salamanca, Spain. There are $10 \mathrm{~Hz}$ PW and multi PW lasers under construction or under design in several places. Among them, particularly advanced are the three pillars of ELI [14].

The characteristics of the Salamanca VEGA laser can be found in Table 1. It is a Ti:Sapphire laser working at a central wavelength close to $800 \mathrm{~nm}$ and with pulse durations of $30 \mathrm{fs}$. VEGA has a common front end (see Fig. 1) and three lines, VEGA-1 arriving to $20 \mathrm{TW}$ at 10 $\mathrm{Hz}$, VEGA-2 arriving at $200 \mathrm{TW}$ at $10 \mathrm{~Hz}$, and the PW, VEGA-3, at only one $\mathrm{Hz}$, i.e. at one shot per second. The compressor for VEGA-3 is shown in Fig. 2, it is a typical four grating compressor arranged in two levels.
At low powers $(\mathrm{GW}) \mathrm{kHz}$ repetition rates are easy to obtain and moreover one can work in air in several cases.

For example Fig. 4 shows a set-up developed at Salamanca to generate soft X-rays (tens of $\mathrm{keV}$ ) with a 120 fs Ti:Sapphire laser delivering pulses of only a few milijoules per shot. In that case we focused the laser on a thick copper plate, as indicated in Fig. 4, using a microscope objective (and therefore a very wide numerical aperture). Ionization releases electrons that move back and forth driven by the laser electric field oscillation and that when colliding back to the copper plate generate Bremsstrahlung X-rays.

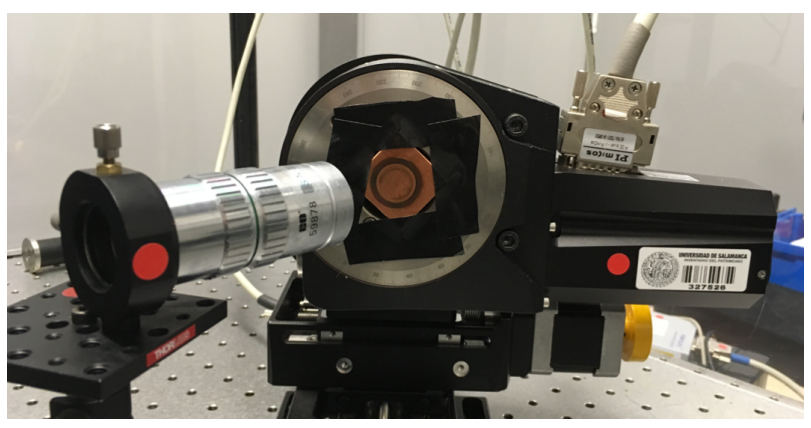

Fig. 4. CLPU soft X-ray system using a multi-GW laser and working in air. The system runs at $\mathrm{kHz}$ repetition rate. Unfortunately this easy geometry is not efficient at higher intensities.

\section{Targetry}

Assuming that there are a few $1 \mathrm{PW} / 1 \mathrm{~Hz}$ lasers ready to fire for long times, then is the problem to find targets for them. And this is a major bottleneck. Of course, the targets depend on the experiments we plan to perform with the laser. The most common uses of the high intensity lasers are acceleration (electrons, protons or ions), warm dense plasmas, and betatron radiation. In some case the accelerated beams or the generated radiation are used in turn as secondary sources (for example, neutrons obtained from accelerated protons).

For electron acceleration studies, the problem is a bit easier. Underdense (underdense relative to the $1.5 \mathrm{eV}$ photons of the near infrared lasers as Ti:Sapphire) gas targets are possible and so the replacement of the gas is straightforward. However for Laser Wakefield Acceleration (LWFA) and related techniques it is necessary to reach certain density of the gas. A pulsed valve is a good choice at low repetition rate but problematic beyond $0.1 \mathrm{~Hz}$. Moreover shooting so quickly implies a large amount of gas introduced in the chamber and this has to be carefully engineered. Fortunately, typically electron experiments use long focal lengths, long Rayleigh lengths and so are not so exigent with the longitudinal position of the target. Some acceleration approaches beyond the $\mathrm{GeV}$ require hollow fibers and the transverse alignment is critical.

Proton and ion acceleration have slightly different requirements. For TNSA (Target Normal Sheath 
Acceleration) studies thin targets with overcritical or at least near critical densities are necessary. Moreover TNSA is not possible with thick targets. The mechanism requires at least targets of 1 to 10 micrometer thick and works even better with submicron thick targets. Preparing one thin target is not complicated, but this represents one shot. The next approach is to prepare a set of targets (a plate with a number of targets), a carousel or other techniques. Each laser shot hits (and destroys) one of the targets. Between shot and shot the target holder moves and positions the next one (and this implies typically recheck of the focus position). All that is easy at single shot, possible at few shots per minute, but impossible at $\mathrm{Hz}$ or beyond. So it is necessary to find other strategies.

As a result of this targetry is a very active field now inside the high intensity laser community. Some approaches are very interesting, as the idea developed by CEA Grenoble of continuous production of a thin ribbon of solid hydrogen [15], obviously at very high pressure and extremely low temperature. However several laser teams would like to study this interaction with pure targets. In this context, the low temperature laboratory, at CEA-Grenoble has developed a cryostat able to continuously produce a thin hydrogen ribbon (50 and 100 micrometer thick). A new extrusion concept, without any moving part has been carried out, using only the thermodynamic properties of the fluid. This implies state of the art techniques in cryogenic cooling. Besides the use of pure hydrogen, that guarantees the existence of protons in the plasma, the use of pure hydrogen has other advantages and peculiarities: it is a pure atomic species, so there is no influence of other ions; the plasma densities are due to the electronic densities (electrons per cubic centimeter) and in this case the density of this solid hydrogen plus the fact that each atom has only one electron constitute an experimental scenario a bit different than the one with $\mathrm{Al}$ foils (where protons come from adsorbed hydrogen atoms and water molecules) and hydrocarbon plastic sheet products made from the resin Polyethylene Terephthalate (Mylar) where besides water the same molecule has hydrogen.

The scenario for such targets is more complicated with recent studies that show that proton/ion acceleration is more efficient with structured targets [16]. Mass production of those targets is possible if they provide to be efficient enough, but it is going to be expensive and time consuming.

Solid targets are not the only possibility to work with plasma densities critical or near-critical. One alternative is to work with high-pressure gases and with specifically designed nozzles that allow a high gas density a few millimeters away from the nozzle [17]. We stress on the need to have the nozzle away from the laser focal spot in order to get a reasonable lifetime of the nozzle. Gas clusters generated by the expansion of a gas jet plume into vacuum are also used [18].

Another interesting approach to continuous targets is the possibility of specifically designed liquid targets. The idea of using a liquid jet is not new in the laser community. For example remember the old times of the dye lasers. However in this case there are several extra considerations. The first one is the need of very small sources. The second one is the contamination generated by the liquids. Regarding the size of the liquid jet, nozzle technology has advanced dramatically during the last decades and now liquid columns comparable to the focal spot are possible (see Fig 5). Also the column can split in micro-droplets with the help of a piezoelectric attached at the nozzle tip. This also allows synchronization between the droplets and the laser pulses. The most remarkable evolution of the micro-jets is the possibility to make a controlled collision of two micro-jets to get a flat liquid curtain conveniently thin for proton acceleration (see Fig 6).

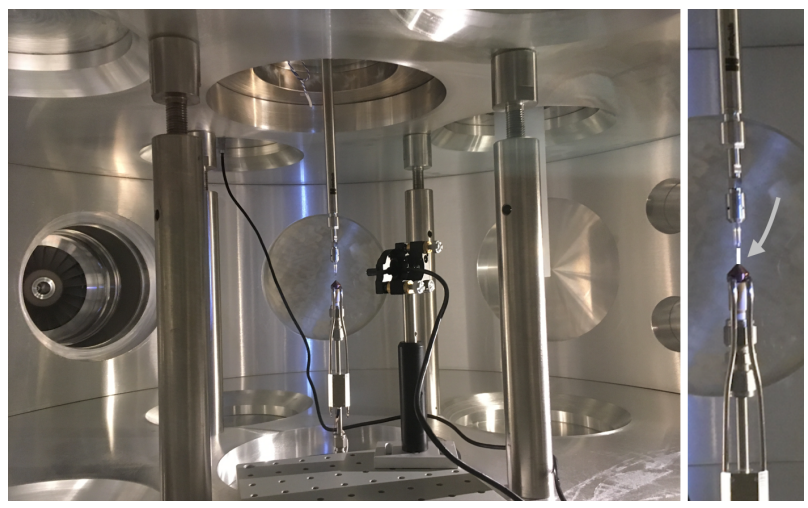

Fig. 5. CLPU liquid column set-up. General view (right) and zoom of the detail of the liquid column (left). The liquid column itself is too narrow (well bellow 100 microns) to bee seen in the picture and has been highlighted (it is indicated by the arrow at the left).

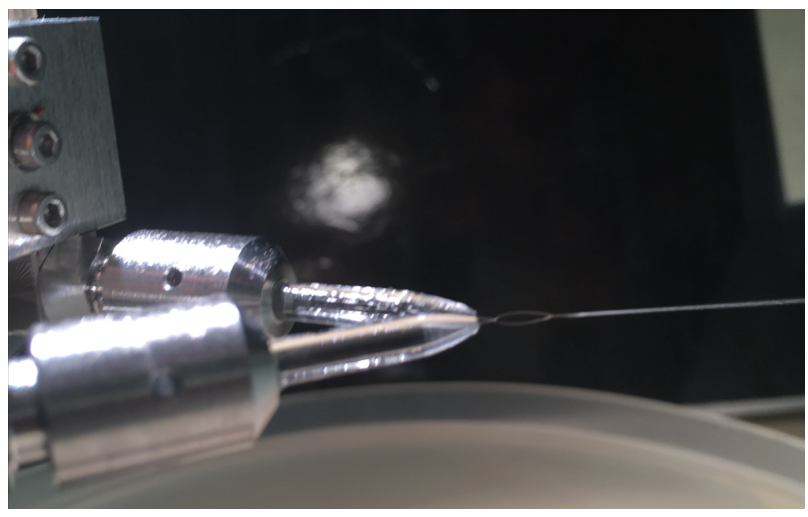

Fig. 6. CLPU flat liquid curtain made by the precise collision of two jets. Observe that all that has to be operative at vacuum and compatible with the low pressure $\left(10^{-6} \mathrm{mbar}\right)$ and therefore a catcher (not shown in the figure) to collect the final jet and cold finger to collect the vaporized liquid are fundamental.

\section{Additional problems}

Working at high repetition rates, debris from the target is a fundamental problem that needs to be analyzed carefully before any experiment. The situation is quite different with liquids than with solids.

Micro liquid jets are a promising technology for continuous reliable targets. However there are several 
problems related to the targets that are far from being solved, the most relevant one and specific for liquids is the extraction of the unused liquid. For this purpose it is necessary to prepare a catcher that gets the remainder of the liquid column. The catcher can be seen in Figure 6, in the zoom at right. The catcher extracts the liquid column. Moreover liquids can evaporate quite quickly in vacuum at room temperature. So it is necessary to place a cold finger close to the jet to trap most of the vaporized part.

When considering solid targets, nano-particle or microparticle debris can not move too far and probably are not a big deal with the gratings or other optical components provided that the ventilation of the vacuum lines is planned in the correct way (ventilation procedures are not always considered carefully enough). Such solid targets are a problem only for the focusing parabolas (for that reason in many labs the cleaning or replacement of the main parabola has to be done quite often). The damage of the parabola can be minimized placing a pellicle in front of it.

Also need to comment on another problem specifically relevant at high repetition rates: shock waves. At one $\mathrm{Hz}$, or more, the shock waves that one shot generates can affect the next one. Shock waves are very relevant at PW or multi-PW laser shots. They generate shock waves that can damage nozzles or other key components of the continuous flux systems. Moreover, in high repetition rate, one shock can feel the target still modified by the previous pulse.

Target composition is not regarded in general as a hazard for the laser longevity at high repetition rates, but it must be carefully considered. Most experimental setups work in high vacuum (typically in the 10-6 millibar region) and -what is more restrictive- free of hydrocarbons. Released hydrocarbons can move across the vacuum space that is generally connected to the gratings. The high fluence of the laser beam allows polymerization at the surface of the optical components, and this is particularly dangerous for the gratings. Obviously this is a noticeable at high repetition rates. The effect is the same that appears on some femtosecond oscillators working at high fluences, as the case of certain CEP (Carrier Envelope Phase) oscillators.

A last point to comment is radiation shielding. Radiation is generally proportional to the number of shots, so multiplying the repletion rate multiplies the radiation shielding problems, and the experiments need quite heavy bunkers to shield the ionizing radiation generated. Fortunately not all acceleration experiments generate the same amount of radiation. And a balance between different experiments has to be carefully planned.

In principle, it could be possible to work in air also. The fluences involved are much bigger that the critical power for the onset of filamentation. So a PW in air will filament and because of the intensity clamping, the beam is going to break in multiple filaments.

\section{Vacuum polarization}

Let's conclude with an optimistic message. Besides all those experiments where targets represent a bottleneck, there is a family of very relevant experiments that do not require a target: they just require vacuum. Therefore those experiments can take full advantage of the multiPW multi-Hz lasers on construction now.

There are two big families of experiments in the optical domain (infrared, visible or ultraviolet). One is the nonlinear propagation in vacuum. Oversimplifying, the experiment consists in focusing such a big laser arriving to a high intensity at vacuum and then studying the propagation of the beam after the focus to detect small deviations from the linear regime.

The other family of experiments is the collision of two laser beams. Photon-photon cross section in the optical regime is extraordinarily small (as can be seen in Fig. 7). Therefore the way to get a measurable signal is to prepare the collision of two big laser pulses [19]. There is no target so the experiment can run using the laser full repetition rate. With multi $\mathrm{PW}$ we are still far, too far, from the Schwinger limit, as a consequence the possibilities to get a collision are very small and require long measuring times (i.e. many laser shots). On the contrary, this can provide a direct measure on the heart of QED (Quantum Electro-Dynamics). Direct information on the existence or not of axions can be obtained from such experiments.

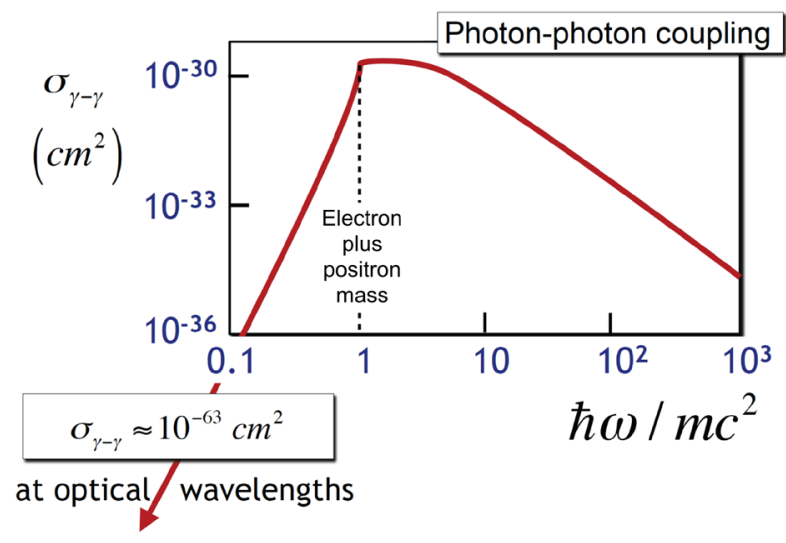

Fig. 7. Photon-photon cross section versus photon energy (for two photons of the same energy) versus the photon energy (in units of the electron mass, $m c^{2}$ ). The photon-photon coupling is extraordinarily small when one considers infrared photons. However, the direct study of such coupling making "collisions" of two big lasers can be of paramount relevance in theoretical physics.

\section{Conclusions}

In conclusion, with today's available, or almost ready, high repetition rate lasers we can -we must- plan a new generation of experiments able to take profit of those relevant laser tools. Among those experiments, particle acceleration, warm dense matter, betatron radiation, etc., are relevant. But also quantum vacuum experiments have to be considered in order to get a fundamental step 
forward on the laser implications on technology and fundamental physics

Support from Spanish Ministerio de Economía, Industria y Competitividad (MINECO) through the PALMA Grant No. FIS2016-81056-R, from LaserLab Europe IV Grant No. 654148, and from Junta de Castilla y León Grant No. CLP087U16 is acknowledged. We particularly acknowledge an equipment grant PUU15-EE-3784 from MINECO that funded the CLPU liquid target system.

\section{References}

1. D. Strickland and G. Mourou, Optics Communications 56, 219 (1985)

2. P. Maine, D. Strickland, P. Bado, M. Pessot and G Mourou, IEEE Journal on Quantum Electronics 24 398 (1988)

3. E. B. Treacy, IEEE J. Quant Electron 5 (1969) 454

4. O. E. Martinez, J. P. Gordon and R. L. Fork, Journal Optical Society of America A 1, 1003 (1984)

5. https://lasers.1lnl.gov/

6. http://www-lmj.cea.fr/

7. X. Liang, et al, in Laser Congress 2017 (ASSL, LAC), OSA Technical Digest (online) (Optical Society of America, 2017), paper AW4A.1.

8. Jae Hee Sung, et al., Optics Lett. 42, 2058 (2017)

9. R Lera, PhD Thesis, University of Salamanca, 2018

10. Ch. G. Durfee, Charles et al., Optics Express 20 13677 (2012)

11. http://bella.lbl.gov/

12. https://www.hzdr.de

13. https://www.clpu.es

14. https://eli-laser.eu

15. S. Garcia, D. Chatain, J. Perin, Laser and Particle Beams, 32, 569 (2014)

16. A. Macchi, M. Borghesi, M. Passoni, Reviews of Modern Physics 85, 751 (2013)

17. F. Sylla, et al, Phys. Rev. Lett. 110, 085001 (2013)

18. T. Ditmire, et al, Nature 398, 489 (1999)

19. D. Tommasini, D. Novoa, L. Roso, in $\mathrm{K}$. Yamanouchi et al eds, Progress in Ultrafast Intense Laser Science, Springer Series in Chemical Physics, vol 106 (2014), Springer 